\title{
Willingness of the General Public to Receive A COVID-19 Vaccine Booster - China, April-May 2021
}

\author{
Xiaoxiao Wang ${ }^{1, \&} ;$ Leyuan $\mathrm{Liu}^{2, \&} ;$ Minyue Pei ${ }^{1} ;$ Xiaoguang $\mathrm{Li}^{2, * \#}$ Nan $\mathrm{Li}^{1, *}$
}

\begin{abstract}
Summary
What is already known about this topic?

A coronavirus disease 2019 (COVID-19) vaccine booster is planned for administration to eligible individuals. Understanding the factors that influence attitudes towards the booster shot will help to identify groups that will most readily accept a booster dose.

What is added by this report?

Of the individuals polled, $75.2 \%$ reported they would receive a booster shot. Sociodemographic characteristics influencing booster vaccine acceptance included age, gender, occupation, and education. Moreover, those who had been vaccinated against influenza, who believed herd immunity would be effective against severe acute respiratory syndrome coronavirus 2 , and who reported reduced anxiety after vaccination were more likely to accept a booster dose.

What are the implications for public health practice?

A booster shot of the COVID-19 vaccine could be widely accepted. Communicating about the effectiveness of the COVID-19 vaccine and the impact of infection on people's health could help increase public willingness to get a booster dose.
\end{abstract}

On October 11, 2021, the strategic advisory group of experts (SAGE) of the World Health Organization (WHO) recommended that an additional dose of coronavirus disease 2019 (COVID-19) vaccine should be offered to moderately and severely immunocompromised people and to those aged 60 and over who were previously immunized with Sinovac or Sinopharm inactivated vaccines (1). The National Health Commission of the People's Republic of China (NHC) and the United States Centers for Disease Control Advisory Committee on Immunization Practices (ACIP) are planning a COVID-19 vaccine booster so that vaccinated people can maintain protection over the coming months (2-3). Numerous studies have shown that vaccination willingness is influenced by a variety of factors and that it changes over time (4-5). It is necessary to understand the public's willingness to receive a COVID-19 vaccine booster. Identifying factors that influence booster vaccine acceptance will aid in determining who is most likely to accept a booster dose.

Online questionnaires were completed by 2,047 vaccinated Chinese adults in April and May 2021. Respondents' sociodemographic characteristics, attitudes towards vaccination, and attitudes towards a COVID-19 vaccine booster were collected. All data were analyzed using $\mathrm{R}$ statistical software (version 4.0.3, R Core Team, Vienna, Austria). Logistic regression models were built to identify factors associated with respondents' acceptance of a COVID19 vaccine booster. The odds ratio (OR) and its corresponding $95 \%$ confidence interval (CI) were calculated.

Of the respondents, $75.2 \%$ reported they planned to receive a booster shot. Respondents who expressed significantly higher acceptance of a booster dose tended to be aged 25-54 years old, male, non-healthcare workers, and less educated. Moreover, those who had been vaccinated against influenza $(\mathrm{OR}=1.26,95 \% \mathrm{CI}$ : 1.01-1.57), who believed herd immunity would be effective against severe acute respiratory syndrome coronavirus 2 (SARS-CoV-2) infection $(\mathrm{OR}=3.58$, 95\% CI: 2.69-4.77), or who reported reduced anxiety after vaccination $(\mathrm{OR}=1.27,95 \% \mathrm{CI}: 1.02-1.59)$ were more likely to report planning to receive a booster dose.

Based on these results, it seems that a booster shot of the COVID-19 vaccine could be widely accepted in China. Communicating to the public the effectiveness of COVID-19 vaccines and the impact of COVID-19 infection on one's health could increase individuals' willingness to receive a booster dose.

In April and May 2021, an online questionnaire was disseminated via WeChat, a Chinese multipurpose social media app. Using WeChat moments, which spread questionnaires by snowballing, 1,656 respondents were recruited. To reduce the risk of bias due to starting with a single sample source, 403 
additional respondents were recruited via the Tencent questionnaire sample database. This database contains over 1 million people with verified personal information, and we used the recruitment service to recruit subjects aged $\geq 18$. Incomplete questionnaires were excluded. The final sample consisted of 2,047 respondents. Respondents' sociodemographic characteristics, flu vaccination history, attitudes towards herd immunity, anxiety levels after initial vaccination, acceptance of a booster shot, and antibody tests were collected. The study was approved by Peking University Third Hospital Medical Science Research Ethics Committee (No. 2021-184-01).

The age- and gender-standardized acceptance rate of a COVID-19 vaccine booster was calculated using the 2010 population census of China as the reference ( 6 ). Logistic regression models were built to identify factors influencing COVID-19 vaccine booster acceptance (event: receiving COVID-19 vaccine booster when available). All data were analyzed using $\mathrm{R}$ statistical software. A $P$-value $<0.05$ was considered statistically significant.

Of the 2,047 vaccinated respondents (Table 1), $1,540(75.2 \%)$ reported that they planned to receive a COVID-19 vaccine booster shot when it was available. The age- and gender-standardized acceptance rate was $75.8 \%$ (Table 2 ). In addition, 1,257 (81.6\% of those who planned to receive the booster shot) reported that they would receive antibody tests, which could help to determine the effectiveness of the booster dose.

COVID-19 vaccine booster acceptance rate was highest among adults aged $45-54$ years $(81.2 \%)$ and lowest among adults aged $\geq 65$ years $(69.6 \%)$. Male respondents were more likely than female respondents to accept a COVID-19 vaccine booster $(80.2 \%$ vs. $72.2 \%)$, and those who were not healthcare workers were more likely to accept a booster dose than healthcare workers $(79.3 \%$ vs. $67.0 \%)$. Respondents who held a bachelor's degree or below were more likely to accept a booster dose $(68.9 \%, 74.5 \%, 83.3 \%$, vs. $85.4 \%)$. Vaccine booster acceptance was slightly higher among respondents who earned $<5,000 \mathrm{CNY}$ per month $(77.7 \%$ vs. $73.6 \%)$. Respondents who lived in rural areas were more likely to accept a booster dose $(83.1 \%$ vs. $74.1 \%)$. Moreover, those who had been vaccinated against influenza $(77.7 \%$ vs. $73.4 \%$ who had not been), who believed herd immunity would be effective against SARS-CoV-2 (78.9\% vs. $47.5 \%$ who did not believe this), and who reported reduced anxiety after vaccination $(77.0 \%$ vs. $71.7 \%$ who did not report this) were more likely to accept a booster dose.
TABLE 1. Characteristics of the study population.

\begin{tabular}{|c|c|c|}
\hline Characteristics & $\begin{array}{c}\text { WeChat sample } \\
(n=1,644)\end{array}$ & $\begin{array}{c}\text { Tencent sample } \\
(n=403)\end{array}$ \\
\hline \multicolumn{3}{|l|}{ Age group, years } \\
\hline $18-24$ & $184(11.3)$ & $14(3.5)$ \\
\hline $25-34$ & $614(37.6)$ & $18(4.5)$ \\
\hline $35-44$ & $451(27.6)$ & $43(10.7)$ \\
\hline $45-54$ & $254(15.5)$ & $245(60.8)$ \\
\hline $55-64$ & $91(5.6)$ & $68(16.9)$ \\
\hline$\geq 65$ & $41(2.5)$ & $15(3.7)$ \\
\hline \multicolumn{3}{|l|}{ Gender } \\
\hline Male & $613(37.3)$ & $164(40.7)$ \\
\hline Female & $1,031(62.7)$ & $239(59.3)$ \\
\hline \multicolumn{3}{|l|}{ Non-healthcare staff } \\
\hline Yes & $973(59.2)$ & $398(98.8)$ \\
\hline No & $671(40.8)$ & $5(1.2)$ \\
\hline \multicolumn{3}{|l|}{ Education } \\
\hline $\begin{array}{l}\text { Junior high school and } \\
\text { below }\end{array}$ & $105(6.4)$ & $135(33.5)$ \\
\hline Senior high school & $78(4.7)$ & $216(53.6)$ \\
\hline Associate or bachelor & $809(49.2)$ & $49(12.2)$ \\
\hline Master and above & $652(39.7)$ & $3(0.7)$ \\
\hline \multicolumn{3}{|l|}{ Income (CNY per month) } \\
\hline $0-2,000$ & $201(12.2)$ & $106(26.3)$ \\
\hline $2,000-5,000$ & $300(18.3)$ & $221(54.8)$ \\
\hline $5,000-10,000$ & 459 (27.9) & $66(16.4)$ \\
\hline 10,000 and above & $684(41.6)$ & $10(2.5)$ \\
\hline \multicolumn{3}{|l|}{ Area type } \\
\hline Rural & $1,498(91.6)$ & $285(70.9)$ \\
\hline Urban & $138(8.4)$ & $117(29.1)$ \\
\hline \multicolumn{3}{|l|}{ Flu vaccination history } \\
\hline Yes & $680(41.4)$ & $189(46.9)$ \\
\hline No/unsure & $964(58.6)$ & $214(53.1)$ \\
\hline \multicolumn{3}{|l|}{$\begin{array}{l}\text { Whether herd immunity } \\
\text { works }\end{array}$} \\
\hline Yes & $1,423(86.6)$ & $382(94.8)$ \\
\hline No/unsure & $221(13.4)$ & $21(5.2)$ \\
\hline \multicolumn{3}{|l|}{$\begin{array}{l}\text { Whether vaccination help } \\
\text { reduce anxiety }\end{array}$} \\
\hline Yes & $1,098(66.8)$ & $268(66.5)$ \\
\hline No & $546(33.2)$ & $135(33.5)$ \\
\hline
\end{tabular}

Note: All data are described in term of "Number (\%) of participants". Abbreviation: $\mathrm{CNY}=$ China Yuan.

The multiple logistic regression model identified the people most likely to get the booster dose as soon as it was available (Table 3). Those who expressed significantly higher acceptance of a booster dose included respondents who were: 25-34 years old 
TABLE 2. The process of calculating age- and gender- standardized acceptance rate of a COVID-19 vaccine booster.

\begin{tabular}{llccc}
\hline Age (year) & Gender & Observed acceptance rates (\%) & Population according to census 2016 & Expected number \\
\hline $18-24$ & Female & 71.0 & $83,878,762$ & $59,553,921$ \\
& Male & 77.0 & $85,832,496$ & $66,091,022$ \\
$25-34$ & Female & 69.0 & $97,793,195$ & $67,477,305$ \\
& Male & 76.4 & $100,358,860$ & $76,674,169$ \\
$35-44$ & Female & 73.1 & $118,780,141$ & $86,828,283$ \\
& Male & 80.5 & $123,999,782$ & $99,819,825$ \\
$45-54$ & Female & 78.3 & $90,208,072$ & $70,632,920$ \\
& Male & 86.2 & $94,139,652$ & $81,148,380$ \\
$55-64$ & Female & 67.7 & $69,062,392$ & $46,755,239$ \\
& Male & 81.8 & $70,917,364$ & $58,010,404$ \\
\multirow{2}{*}{ T65 } & Female & 60.6 & $49,507,029$ & $30,001,260$ \\
& Male & 82.6 & $48,430,783$ & $40,003,827$ \\
\hline
\end{tabular}

Note: The age- and gender- standardized acceptance rate of a COVID-19 vaccine booster equals $75.8 \%$.

Abbreviation: COVID-19=coronavirus disease 2019.

$(\mathrm{OR}=2.06,95 \%$ CI: 1.09-3.91); 35-44 years old $(\mathrm{OR}=2.24$, 95\% CI: 1.18-4.28); 45-54 years old $(\mathrm{OR}=2.07,95 \%$ CI: $1.09-3.94)$; male $(\mathrm{OR}=1.33,95 \%$ CI: $1.05-1.67) ;$ and non-healthcare workers $(\mathrm{OR}=1.50, \quad 95 \% \quad \mathrm{CI}: 1.17-1.92)$. Booster shot acceptance was also higher among those who had: a junior high school level of education or below (OR=2.64, 95\% CI: 1.50-4.62); a high school level of education $(\mathrm{OR}=2.12,95 \% \mathrm{CI}$ : $1.34-3.35)$; and who had been vaccinated against influenza $(\mathrm{OR}=1.26,95 \%$ CI: 1.01-1.57). Finally, those who believed herd immunity would be effective against SARS-CoV-2 or who reported reduced anxiety after vaccination were more likely to accept a booster dose $(\mathrm{OR}=3.58,95 \%$ CI: $2.69-4.77$ and $\mathrm{OR}=1.27,95 \%$ CI: $1.02-1.59$, respectively).

\section{DISCUSSION}

This survey demonstrated that most of the vaccinated respondents $(75.2 \%)$ would accept a COVID-19 vaccine booster shot when it became available. Although data on the efficacy and safety of the booster shot are still lacking, booster shots have a higher level of acceptance now than earlier doses of the vaccines did (4). Multiple studies have shown that vaccine acceptance changes over time, and the proportion of people who accept a vaccine may rise as the pandemic continues to fluctuate and the safety of vaccines is properly reported (4-5). Sociodemographic characteristics were important factors affecting the acceptability of the COVID-19 vaccine booster. Respondents who were aged 25-54 years old, male, non-healthcare workers, and less educated expressed significantly higher acceptance of the booster dose. According to WHO recommendations, people aged 60 or older who received inactivated vaccines should receive booster shots. The relatively low acceptance among people over 60 is therefore of great concern.

Moreover, those who had been vaccinated against influenza $(\mathrm{OR}=1.26,95 \% \mathrm{CI}: 1.01-1.57)$, who believed herd immunity would be effective against SARS-COV-2 (OR=3.58, 95\% CI: 2.69-4.77) or who reported reduced anxiety after vaccination $(\mathrm{OR}=1.27$, 95\% CI: 1.02-1.59) were more likely to accept a booster dose. These results suggest that strong confidence in the vaccines would lead to more people getting vaccinated, which is consistent with previous studies (7-8). Therefore, efforts focused on clearly communicating to the public the effectiveness and safety of the COVID-19 booster vaccination and the risk of getting sick and dying from COVID-19 could help increase public willingness to get vaccinated.

This is the first study to address public acceptance of a booster shot of COVID-19 vaccines, and we find that they are likely to be widely accepted. This survey identifies priority groups to target for COVID-19 vaccine booster shots, which is of crucial importance in public health policy implementation (9).

This study was subject to some limitations. First is that convenience sampling was used, which may affect the representativeness of the individuals sampled compared to the population as a whole. The epidemic 
TABLE 3. Influencing factors on COVID-19 vaccine booster preference.

\begin{tabular}{|c|c|c|c|c|c|c|}
\hline \multirow{2}{*}{ Characteristics } & \multicolumn{2}{|c|}{ COVID-19 vaccinebooster preference } & \multicolumn{2}{|c|}{ Univariate analysis } & \multicolumn{2}{|c|}{ Multivariate analysis } \\
\hline & No (percentag & s (percentage \%) & $\begin{array}{c}\text { Crude OR } \\
(95 \% \mathrm{Cl})\end{array}$ & $\boldsymbol{P}$ & $\begin{array}{c}\text { Adjusted OR } \\
(95 \% \mathrm{Cl})\end{array}$ & $\boldsymbol{P}$ \\
\hline \multicolumn{7}{|l|}{ Age group, years } \\
\hline $18-24$ & $53(26.8)$ & $145(73.2)$ & $1.19(0.62,2.29)$ & 0.596 & $1.99(0.97,4.10)$ & 0.062 \\
\hline $25-34$ & $178(28.2)$ & $454(71.8)$ & $1.11(0.61,2.02)$ & 0.727 & $2.06(1.09,3.91)$ & 0.026 \\
\hline $35-44$ & $119(24.1)$ & $375(75.9)$ & $1.37(0.75,2.52)$ & 0.304 & $2.24(1.18,4.28)$ & 0.014 \\
\hline $45-54$ & $94(18.8)$ & $405(81.2)$ & $1.88(1.02,3.46)$ & 0.044 & $2.07(1.09,3.94)$ & 0.026 \\
\hline $55-64$ & $42(26.4)$ & $117(73.6)$ & $1.21(0.62,2.37)$ & 0.570 & $1.14(0.56,2.30)$ & 0.719 \\
\hline$\geq 65$ & $17(30.4)$ & $39(69.6)$ & Ref. & & Ref. & \\
\hline Gender & & & & $<0.001$ & & 0.016 \\
\hline Male & $154(19.8)$ & $623(80.2)$ & $1.56(1.26,1.93)$ & & $1.33(1.05,1.67)$ & \\
\hline Female & $353(27.8)$ & $917(72.2)$ & Ref. & & Ref. & \\
\hline Non-healthcare staff & & & & $<0.001$ & & 0.002 \\
\hline Yes & $284(20.7)$ & 1,087 (79.3) & $1.88(1.53,2.32)$ & & $1.50(1.17,1.92)$ & \\
\hline No & $223(33.0)$ & $453(67.0)$ & Ref. & & Ref. & \\
\hline \multicolumn{7}{|l|}{ Education } \\
\hline Junior high school and below & $35(14.6)$ & $205(85.4)$ & $2.65(1.79,3.93)$ & $<0.001$ & $2.64(1.50,4.62)$ & 0.001 \\
\hline Senior high school & $49(16.7)$ & $245(83.3)$ & $2.26(1.60,3.20)$ & $<0.001$ & $2.12(1.34,3.35)$ & 0.001 \\
\hline Associate or bachelor & $219(25.5)$ & $639(74.5)$ & $1.32(1.05,1.65)$ & 0.016 & $1.26(0.98,1.63)$ & 0.070 \\
\hline Master and above & $204(31.1)$ & $451(68.9)$ & Ref. & & Ref. & \\
\hline \multicolumn{7}{|l|}{ Income (CNY per month) } \\
\hline $0-2,000$ & $71(23.1)$ & $236(76.9)$ & $1.22(0.89,1.67)$ & 0.220 & $0.64(0.40,1.01)$ & 0.054 \\
\hline $2,000-5,000$ & $114(21.9)$ & $407(78.1)$ & $1.31(1.00,1.71)$ & 0.049 & $0.83(0.59,1.16)$ & 0.269 \\
\hline $5,000-10,000$ & $136(25.9)$ & $389(74.1)$ & $1.05(0.81,1.36)$ & 0.725 & $0.86(0.65,1.15)$ & 0.309 \\
\hline 10,000 and above & $186(26.8)$ & $508(73.2)$ & Ref. & & Ref. & \\
\hline Residence & & & & 0.002 & & 0.553 \\
\hline Rural & $43(16.9)$ & $212(83.1)$ & $1.72(1.22,2.43)$ & & $1.14(0.74,1.76)$ & \\
\hline Urban & $461(25.9)$ & $1,322(74.1)$ & Ref. & & Ref. & \\
\hline Flu vaccination history & & & & 0.028 & & 0.039 \\
\hline Yes & $194(22.3)$ & $675(77.7)$ & $1.26(1.03,1.55)$ & & $1.26(1.01,1.57)$ & \\
\hline No/unsure & $313(26.6)$ & $865(73.4)$ & Ref. & & Ref. & \\
\hline Whether herd immunity works & & & & $<0.001$ & & $<0.001$ \\
\hline Yes & $380(21.1)$ & $1,425(78.9)$ & $4.14(3.14,5.46)$ & & $3.58(2.69,4.77)$ & \\
\hline No/unsure & $127(52.5)$ & $115(47.5)$ & Ref. & & Ref. & \\
\hline Whether vaccination help reduce anxiety & & & & 0.008 & & 0.035 \\
\hline Yes & $314(23.0)$ & $1,052(77.0)$ & $1.33(1.08,1.63)$ & & $1.27(1.02,1.59)$ & \\
\hline No & $193(28.3)$ & $488(71.7)$ & Ref. & & Ref. & \\
\hline
\end{tabular}

Abbreviations: COVID-19=coronavirus disease 2019; OR=odds ratio; $\mathrm{Cl}=$ confidence interval; CNY=China Yuan; Ref.=reference.

was relatively stable when the survey was conducted and therefore not causing a great amount of panic among members of the public, which suggests that this sample provided some insight to public willingness to get a booster dose. Second, acceptance of a COVID-19 vaccine booster could also be influenced by information spread in the media and on social networks, including the local number of daily confirmed cases, the capacity of healthcare services, and relevant policies in different areas. However, we are 
unable to consider these issues in this study due to unavailability of the data. Further investigation is therefore needed in the future.

Conflicts of interest: No conflicts of interest declared.

Funding: Supported by the National Natural Science Foundation of China (Project Number: 81701067, 82101264) and National Major Science and Technology Projects (Project No.2018ZX10 732401-003).

doi: $10.46234 / \mathrm{ccdcw} 2022.013$

* Corresponding authors: Xiaoguang Li, lixiaoguangpuh3@bjmu. edu.cn; Nan Li, linan917@163.com.

\footnotetext{
${ }^{1}$ Research Center of Clinical Epidemiology, Peking University Third Hospital, Beijing, China; ${ }^{2}$ Department of Infectious Diseases, Peking University Third Hospital, Beijing, China.

\& Joint first authors.
}

Submitted: October 13, 2021; Accepted: November 06, 2021

\section{REFERENCES}

1. World Health Organization. SAGE October 2021 meeting highlights. https://www.who.int/news/item/10-10-2021-sage-october-2021-meet ing-highlights. [2021-10-27].
2. Centers for Disease Control and Prevention. COVID-19 vaccine booster shot. https://www.cdc.gov/coronavirus/2019-ncov/vaccines/booster-shot. html. [2021-10-27].

3. National Health Commission of the People's Republic of China. Transcript of press conference of the joint prevention and contro mechanism of the state council on March 28, 2021. http://www.nhc.gov. $\mathrm{cn} / \mathrm{xcs} / \mathrm{s} 3574 / 202103 / \mathrm{b} 8 \mathrm{e} 12 \mathrm{~b} 9385 \mathrm{~b} 44813 \mathrm{af} 117 \mathrm{faad} 928 \mathrm{~b} 7 \mathrm{~d} 3 . \mathrm{shtml}$ [2021-10-27]. (In Chinese).

4. Nehal KR, Steendam LM, Campos Ponce M, van der Hoeven M, Smit GSA. worldwide vaccination willingness for COVID-19: a systematic review and meta-analysis. Vaccines 2021;9(10):1071. http://dx.doi.org/ 10.3390/vaccines 9101071.

5. de Albuquerque Veloso Machado M, Roberts B, Wong BLH, van Kessel $\mathrm{R}$, Mossialos E. The relationship between the COVID-19 pandemic and vaccine hesitancy: a scoping review of literature until August 2021. Front Public Health 2021;9:747787. http://dx.doi.org/10.3389/fpubh.2021. 747787.

6. National Bureau of Statistics of China. Tabulation on the 2010 population census of the People's Republic of China. http://www.stats. gov.cn/tjsj/pcsj/rkpc/6rp/indexch.htm. [2021-10-27]. (In Chinese).

7. Centers for Disease Control and Prevention. Building confidence in COVID-19 vaccines. https://www.cdc.gov/vaccines/covid-19/vaccinatewith-confidence.html. [2021-10-27].

8. Wang Q, Yang LQ, Jin H, Lin L. Vaccination against COVID-19: a systematic review and meta-analysis of acceptability and its predictors. Prev Med 2021;150:106694. http://dx.doi.org/10.1016/j.ypmed.2021. 106694.

9. Han SS, Cai J, Yang J, Zhang JJ, Wu QH, Zheng W, et al. Time-varying optimization of COVID-19 vaccine prioritization in the context of limited vaccination capacity. Nat Commun 2021;12(1):4673. http://dx. doi.org/10.1038/s41467-021-24872-5. 